\title{
Contents
}

\section{Introduction to Acoustics}

Thomas D. Rossing....

1.1 Acoustics: The Science of Sound ................................................. 1

1.2 Sounds We Hear ................................................................... 1

1.3 Sounds We Cannot Hear: Ultrasound and Infrasound .................... 2

1.4 Sounds We Would Rather Not Hear: Environmental Noise Control..... 2

1.5 Aesthetic Sound: Music ........................................................

1.6 Sound of the Human Voice: Speech and Singing .......................... 3

1.7 How We Hear: Physiological and Psychological Acoustics ................. 4

1.8 Architectural Acoustics ............................................................. 4

1.9 Harnessing Sound: Physical and Engineering Acoustics ................... 5

1.10 Medical Acoustics .............................................................. 5

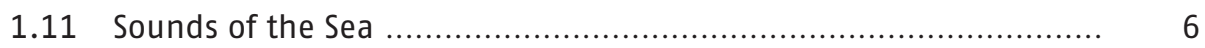

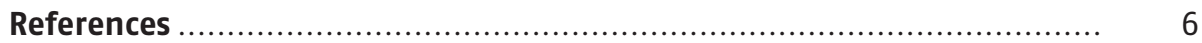

\section{Part A Propagation of Sound}

\section{A Brief History of Acoustics}

Thomas D. Rossing.

2.1 Acoustics in Ancient Times ........................................................ 9

2.2 Early Experiments on Vibrating Strings, Membranes and Plates ...... 10

2.3 Speed of Sound in Air ..................................................... 10

2.4 Speed of Sound in Liquids and Solids ........................................ 11

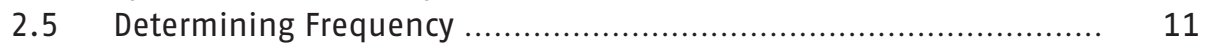

2.6 Acoustics in the 19th Century ................................................ 12

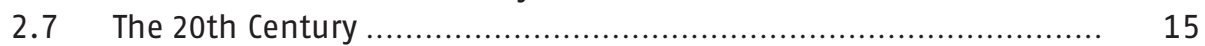

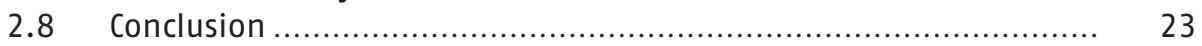

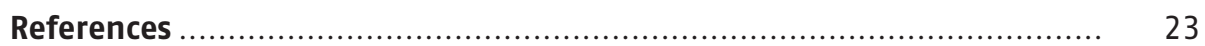

\section{Basic Linear Acoustics}

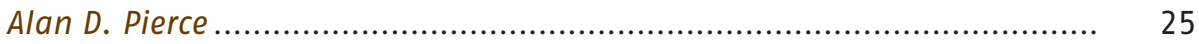

3.1 Introduction .................................................................. 27

3.2 Equations of Continuum Mechanics.......................................... 28

3.3 Equations of Linear Acoustics .................................................. 35

$3.4 \quad$ Variational Formulations ..................................................... $\quad 40$

3.5 Waves of Constant Frequency .................................................. 45

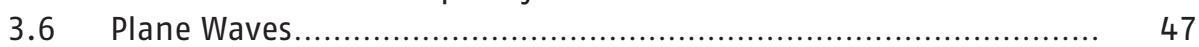


3.7 Attenuation of Sound ....................................................... 49

3.8 Acoustic Intensity and Power .............................................. 58

3.9 Impedance ............................................................. 60

3.10 Reflection and Transmission ............................................... 61

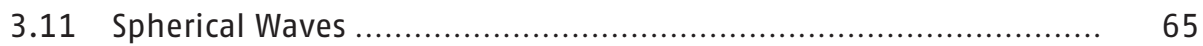

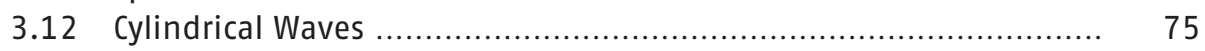

3.13 Simple Sources of Sound ........................................... 82

3.14 Integral Equations in Acoustics ............................................. 87

3.15 Waveguides, Ducts, and Resonators ..................................... 89

3.16 Ray Acoustics ................................................................ 94

3.17 Diffraction ................................................................ 98

3.18 Parabolic Equation Methods ........................................... 107

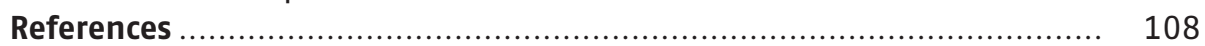

4 Sound Propagation in the Atmosphere

Keith Attenborough ....................................................................... 113

4.1 A Short History of Outdoor Acoustics ...................................... 113

4.2 Applications of Outdoor Acoustics...................................... 114

$4.3 \quad$ Spreading Losses ..................................................... 115

4.4 Atmospheric Absorption............................................ 116

4.5 Diffraction and Barriers ............................................... 116

4.6 Ground Effects .......................................................... 120

4.7 Attenuation Through Trees and Foliage .................................. 129

4.8 Wind and Temperature Gradient Effects on Outdoor Sound ........... 131

4.9 Concluding Remarks ................................................ 142

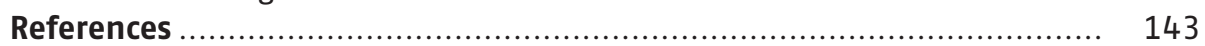

5 Underwater Acoustics

William A. Kuperman, Philippe Roux ........................................ 149

5.1 Ocean Acoustic Environment .................................................. 151

5.2 Physical Mechanisms ......................................................... 155

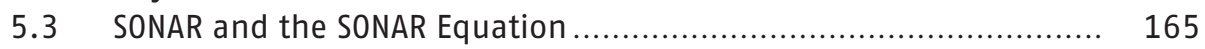

5.4 Sound Propagation Models ............................................ 167

5.5 Quantitative Description of Propagation ................................ 177

5.6 SONAR Array Processing .................................................... 179

$5.7 \quad$ Active SONAR Processing ................................................ 185

5.8 Acoustics and Marine Animals ............................................... 195

5.A Appendix: Units .............................................................. 201

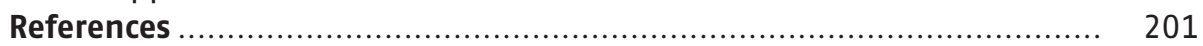

\section{Part B Physical and Nonlinear Acoustics}

\section{Physical Acoustics}

Mack A. Breazeale, Michael McPherson ......................................... 207

6.1 Theoretical Overview ....................................................... 209

6.2 Applications of Physical Acoustics......................................... 219 
$6.3 \quad$ Apparatus .................................................................... 226

6.4 Surface Acoustic Waves ...................................................... 231

$6.5 \quad$ Nonlinear Acoustics ........................................................ 234

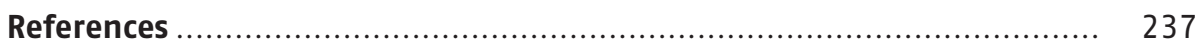

7 Thermoacoustics

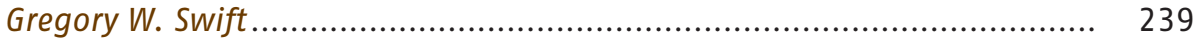

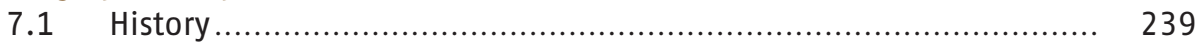

7.2 Shared Concepts ........................................................ 240

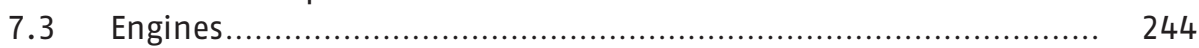

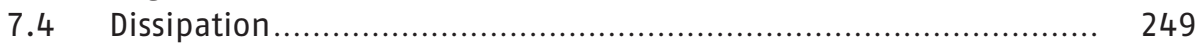

$7.5 \quad$ Refrigeration........................................................ 250

7.6 Mixture Separation ............................................................. 253

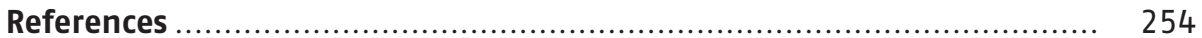

8 Nonlinear Acoustics in Fluids

Werner Lauterborn, Thomas Kurz, Iskander Akhatov............................ 257

$8.1 \quad$ Origin of Nonlinearity ................................................. 258

8.2 Equation of State .......................................................... 259

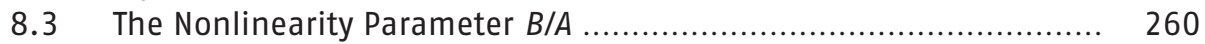

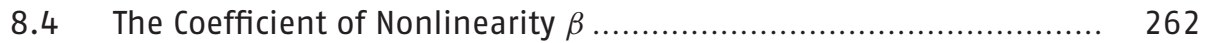

8.5 Simple Nonlinear Waves ............................................... 263

8.6 Lossless Finite-Amplitude Acoustic Waves ................................ 264

8.7 Thermoviscous Finite-Amplitude Acoustic Waves........................ 268

8.8 Shock Waves ............................................................. 271

8.9 Interaction of Nonlinear Waves ......................................... 273

8.10 Bubbly Liquids .......................................................... 275

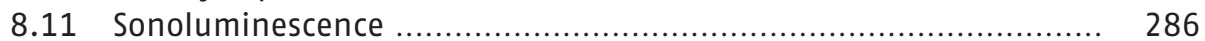

8.12 Acoustic Chaos ............................................................ 289

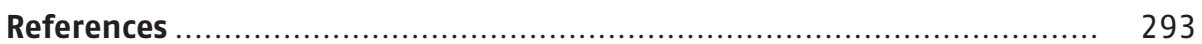

\section{Part C Architectural Acoustics}

\section{Acoustics in Halls for Speech and Music}

Anders Christian Gade............................................................... 301

9.1 Room Acoustic Concepts.................................................. 302

9.2 Subjective Room Acoustics ......................................... 303

9.3 Subjective and Objective Room Acoustic Parameters.................... 306

9.4 Measurement of Objective Parameters................................ 314

9.5 Prediction of Room Acoustic Parameters .................................... 316

9.6 Geometric Design Considerations ......................................... 323

9.7 Room Acoustic Design of Auditoria for Specific Purposes ............... 334

9.8 Sound Systems for Auditoria ............................................ 346

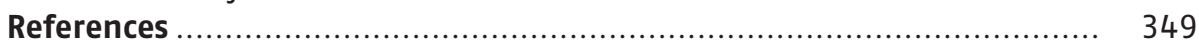


10 Concert Hall Acoustics Based on Subjective Preference Theory

Yoichi Ando

10.1 Theory of Subjective Preference for the Sound Field .................... 353

10.2 Design Studies .............................................................. 361

10.3 Individual Preferences of a Listener and a Performer .................. 370

10.4 Acoustical Measurements of the Sound Fields in Rooms .............. 377

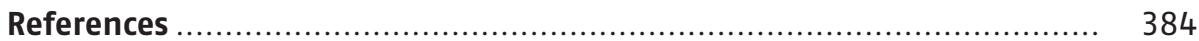

\section{Building Acoustics}

James Cowan.

11.1 Room Acoustics ............................................................ 387

11.2 General Noise Reduction Methods ....................................... 400

11.3 Noise Ratings for Steady Background Sound Levels..................... 403

11.4 Noise Sources in Buildings ................................................. 405

11.5 Noise Control Methods for Building Systems............................ 407

11.6 Acoustical Privacy in Buildings ................................................ 419

11.7 Relevant Standards ................................................... 424

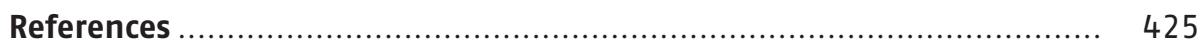

\section{Part D Hearing and Signal Processing}

\section{Physiological Acoustics}

Eric D. Young

12.1 The External and Middle Ear ............................................... 429

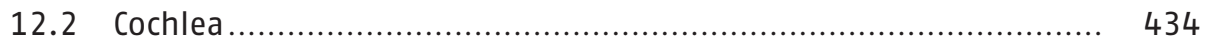

12.3 Auditory Nerve and Central Nervous System ............................. 449

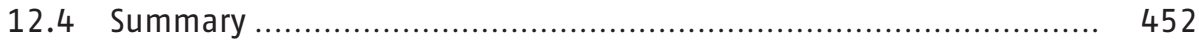

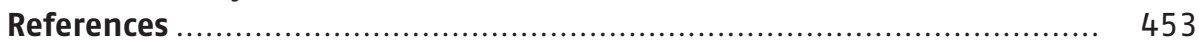

\section{Psychoacoustics}

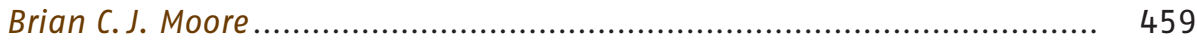

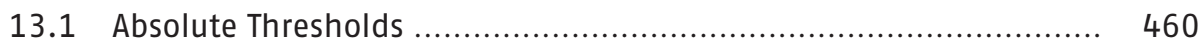

13.2 Frequency Selectivity and Masking ....................................... 461

13.3 Loudness ......................................................................... 468

13.4 Temporal Processing in the Auditory System .............................. 473

13.5 Pitch Perception ........................................................ 477

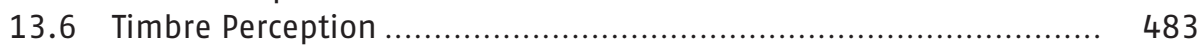

13.7 The Localization of Sounds ............................................... 484

13.8 Auditory Scene Analysis .................................................. 485

13.9 Further Reading and Supplementary Materials ....................... 494

References ................................................................................. 495

\section{Acoustic Signal Processing}

William M. Hartmann ................................................................... 503

14.1 Definitions ................................................................ 504

14.2 Fourier Series .......................................................... 505 


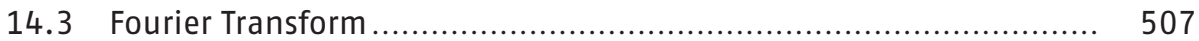

14.4 Power, Energy, and Power Spectrum ..................................... 510

14.5 Statistics ................................................................... 511

14.6 Hilbert Transform and the Envelope ...................................... 514

14.7 Filters ........................................................................... 515

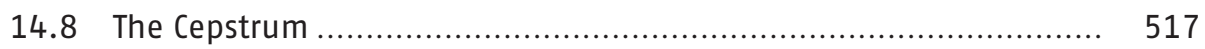

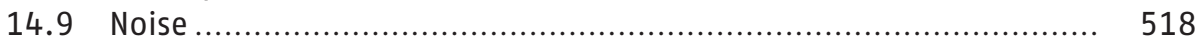

14.10 Sampled data ............................................................... 520

14.11 Discrete Fourier Transform ................................................. 522

14.12 The $z$-Transform ......................................................... 524

14.13 Maximum Length Sequences.......................................... 526

14.14 Information Theory ....................................................... 528

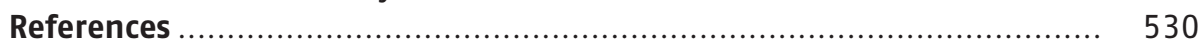

\section{Part E Music, Speech, Electroacoustics}

\section{Musical Acoustics}

Colin Gough

15.1 Vibrational Modes of Instruments ......................................... 535

15.2 Stringed Instruments ...................................................... 554

15.3 Wind Instruments ........................................................... 601

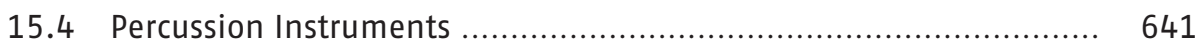

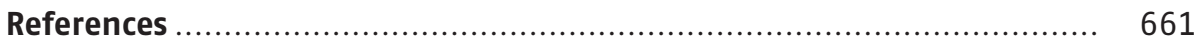

16 The Human Voice in Speech and Singing

Björn Lindblom, Johan Sundberg ...................................................... 669

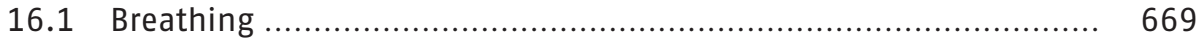

16.2 The Glottal Sound Source ....................................................... 676

16.3 The Vocal Tract Filter ................................................. 682

16.4 Articulatory Processes, Vowels and Consonants ........................ 687

16.5 The Syllable............................................................. 695

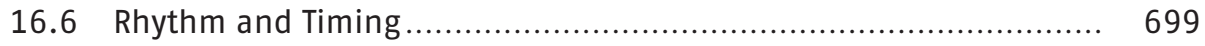

16.7 Prosody and Speech Dynamics ......................................... 701

16.8 Control of Sound in Speech and Singing .................................. 703

16.9 The Expressive Power of the Human Voice ............................... 706

References .............................................................................. 706

\section{Computer Music}

Perry R. Cook .................................................................................. 713

17.1 Computer Audio Basics .............................................. 714

17.2 Pulse Code Modulation Synthesis ................................... 717

17.3 Additive (Fourier, Sinusoidal) Synthesis ................................. 719

17.4 Modal (Damped Sinusoidal) Synthesis ..................................... 722

17.5 Subtractive (Source-Filter) Synthesis....................................... 724

17.6 Frequency Modulation (FM) Synthesis ................................. 727

17.7 FOFs, Wavelets, and Grains ............................................. 728 
17.8 Physical Modeling (The Wave Equation) ................................... 730

17.9 Music Description and Control ............................................... 735

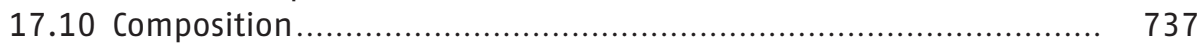

17.11 Controllers and Performance Systems .................................... 737

17.12 Music Understanding and Modeling by Computer ..................... 738

17.13 Conclusions, and the Future .......................................... 740

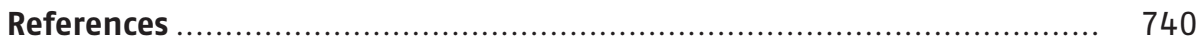

\section{Audio and Electroacoustics}

Mark F. Davis.......................................................................... 743

18.1 Historical Review.............................................................. 744

18.2 The Psychoacoustics of Audio and Electroacoustics .................... 747

18.3 Audio Specifications ..................................................... 751

18.4 Audio Components ...................................................... 757

18.5 Digital Audio ............................................................. 768

18.6 Complete Audio Systems .................................................. 775

18.7 Appraisal and Speculation ................................................. 778

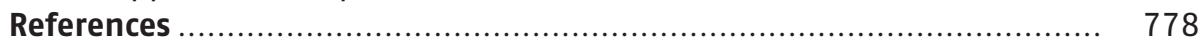

\section{Part F Biological and Medical Acoustics}

\section{Animal Bioacoustics}

Neville H. Fletcher ....................................................................... 785

19.1 0ptimized Communication .................................................. 785

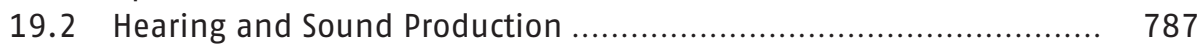

19.3 Vibrational Communication ................................................ 788

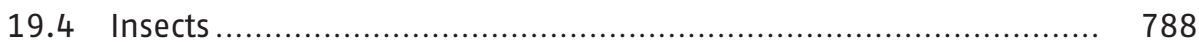

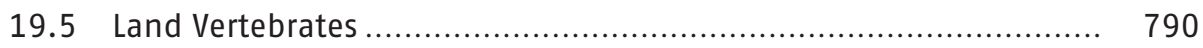

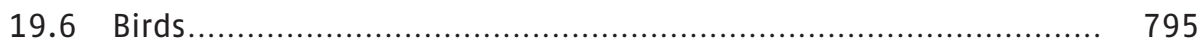

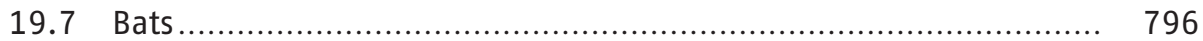

19.8 Aquatic Animals ............................................................. 797

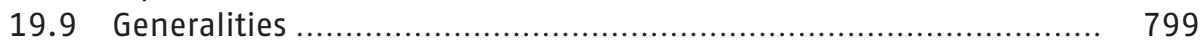

19.10 Quantitative System Analysis........................................... 799

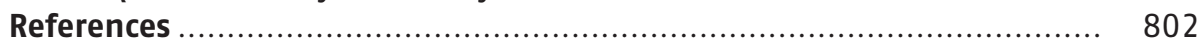

20 Cetacean Acoustics

Whitlow W. L. Au, Marc 0. Lammers ............................................... 805

20.1 Hearing in Cetaceans .................................................... 806

20.2 Echolocation Signals ................................................. 813

20.3 0dontocete Acoustic Communication ..................................... 821

20.4 Acoustic Signals of Mysticetes................................................. 827

20.5 Discussion............................................................. 830

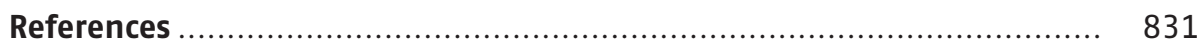

\section{Medical Acoustics}

Kirk W. Beach, Barbrina Dunmire .................................................. 839

21.1 Introduction to Medical Acoustics ........................................... 841 
21.2 Medical Diagnosis; Physical Examination................................... 842

21.3 Basic Physics of Ultrasound Propagation in Tissue ...................... 848

21.4 Methods of Medical Ultrasound Examination ............................ 857

21.5 Medical Contrast Agents ................................................. 882

21.6 Ultrasound Hyperthermia in Physical Therapy ......................... 889

21.7 High-Intensity Focused Ultrasound (HIFU) in Surgery.................. 890

21.8 Lithotripsy of Kidney Stones .............................................. 891

21.9 Thrombolysis................................................................ 892

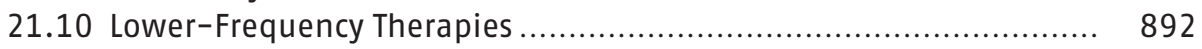

21.11 Ultrasound Safety ......................................................... 892

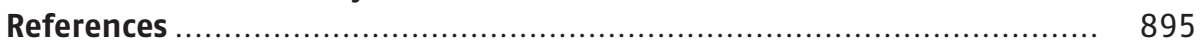

\section{Part G Structural Acoustics and Noise}

\section{Structural Acoustics and Vibrations}

Antoine Chaigne.....

22.1 Dynamics of the Linear Single-Degree-of-Freedom (1-DOF)

Oscillator

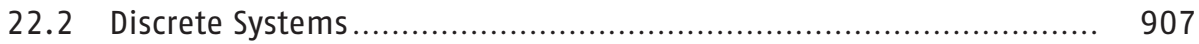

22.3 Strings and Membranes ............................................... 913

22.4 Bars, Plates and Shells....................................................... 920

22.5 Structural-Acoustic Coupling ........................................ 926

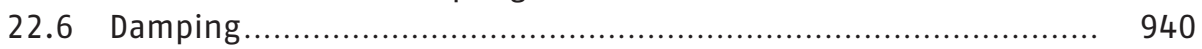

22.7 Nonlinear Vibrations ..................................................... 947

22.8 Conclusion. Advanced Topics........................................... 957

References ............................................................................... 958

\section{Noise}

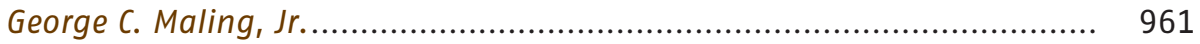

23.1 Instruments for Noise Measurements ....................................... 965

23.2 Noise Sources ............................................................... 970

23.3 Propagation Paths ........................................................... 991

23.4 Noise and the Receiver ............................................... 999

23.5 Regulations and Policy for Noise Control ............................... 1006

23.6 0ther Information Resources.............................................. 1010

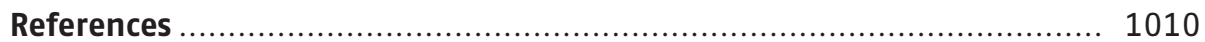

\section{Part H Engineering Acoustics}

\section{Microphones and Their Calibration}

George S. K. Wong.............................................................. 1021

24.1 Historic References on Condenser Microphones and Calibration ...... 1024

24.2 Theory ............................................................... 1024

24.3 Reciprocity Pressure Calibration ......................................... 1026

24.4 Corrections ................................................................ 1029 
24.5 Free-Field Microphone Calibration ........................................ 1039

24.6 Comparison Methods for Microphone Calibration ........................ 1039

24.7 Frequency Response Measurement with Electrostatic Actuators ...... 1043

24.8 Overall View on Microphone Calibration .................................. 1043

24.A Acoustic Transfer Impedance Evaluation ................................. 1045

24.B Physical Properties of Air .............................................. 1045

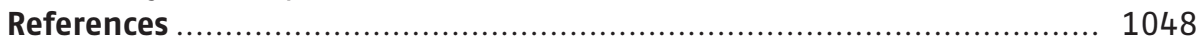

\section{Sound Intensity}

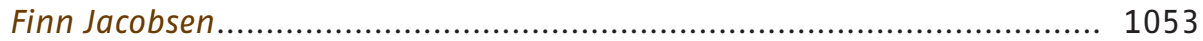

25.1 Conservation of Sound Energy ........................................ 1054

25.2 Active and Reactive Sound Fields ....................................... 1055

25.3 Measurement of Sound Intensity....................................... 1058

25.4 Applications of Sound Intensity ................................... 1068

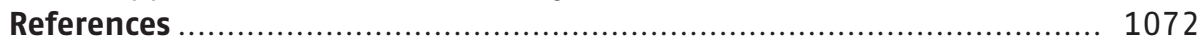

\section{Acoustic Holography}

Yang-Hann Kim .......................................................................... 1077

26.1 The Methodology of Acoustic Source Identification ..................... 1077

26.2 Acoustic Holography: Measurement, Prediction and Analysis ......... 1079

26.3 Summary ................................................................. 1092

26.A Mathematical Derivations of Three Acoustic Holography Methods and Their Discrete Forms................................................... 1092

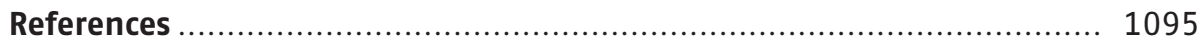

\section{Optical Methods for Acoustics and Vibration Measurements}

Nils-Erik Molin ........................................................................... 1101

27.1 Introduction ........................................................... 1101

27.2 Measurement Principles and Some Applications....................... 1105

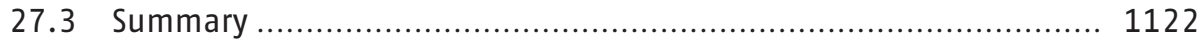

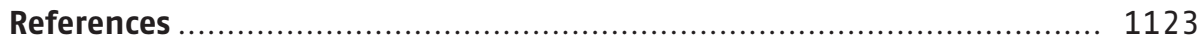

\section{Modal Analysis}

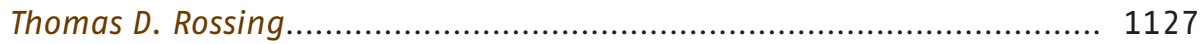

28.1 Modes of Vibration ........................................................... 1127

28.2 Experimental Modal Testing ............................................. 1128

28.3 Mathematical Modal Analysis ........................................ 1133

28.4 Sound-Field Analysis ................................................ 1136

28.5 Holographic Modal Analysis ............................................ 1137

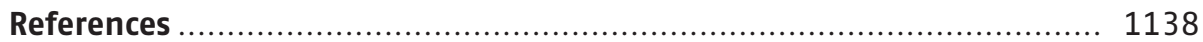

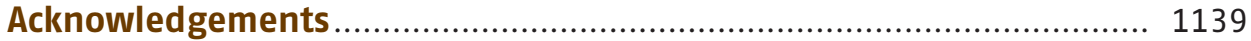

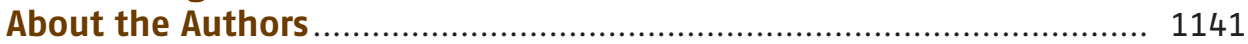

Detailed Contents.................................................................. 1147

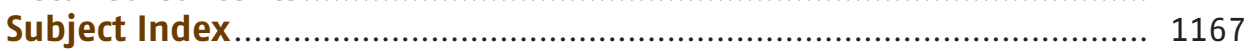

